\title{
Dielectric gels with ultra-high dielectric constant, low elastic modulus, and excellent transparency
}

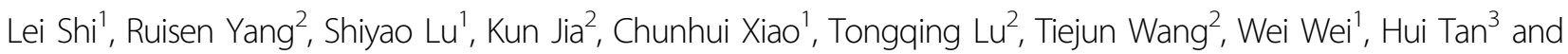
Shujiang Ding ${ }^{1}$

\begin{abstract}
We designed dielectric gels, a new type of polymer-based dielectric material. By using solvents with high dielectric constants, the gels achieve a unique combination of ultra-high dielectric constant, low elastic modulus, and excellent transparency, which are extremely challenging or impossible to realize with traditional polymer dielectrics. The gels exhibit high stretchability (stretch of approximately 10) and low mechanical hysteresis. We demonstrated the use of the dielectric gels by fabricating a bioinspired tunable lens, the focal length of which can be adjusted by varying the applied voltage. We believe that the dielectric gels, as a new type of polymer dielectric, offer new opportunities for soft robotics, sensors, electronics, optics, and biomimetics.
\end{abstract}

\section{Introduction}

As soft and flexible electrical insulators, polymer dielectrics have enabled diverse modern technologies, including electric power systems ${ }^{1}$, flexible electronics ${ }^{2}$, non-volatile memory devices ${ }^{3}$, electrocaloric cooling ${ }^{4}$, and soft robotics ${ }^{5-7}$, benefiting from their diverse functionality. Deformable polymer dielectrics can express mechanical motions in response to electrical stimulation: when a dielectric membrane is subjected to a voltage across its thickness, the membrane suffers an electrostatic force, the so-called Maxwell stress, and thus squeezes in the thickness direction and expands in the area direction. Such dielectrics behave as actuators, as demonstrated by ferroelectric polymers ${ }^{8}$, liquid crystal

Correspondence: Tongqing Lu (tongqinglu@mail.xjtu.edu.cn) or Hui Tan (huitan@email.szu.edu.cn) or Shujiang Ding (dingsj@mail.xjtu.edu.cn) 'Department of Applied Chemistry, School of Science, MOE Key Laboratory for Nonequilibrium Synthesis and Modulation of Condensed Matter, State Key Laboratory for Mechanical Behavior of Materials, Xi'an Jiaotong University, Xi'an 710049, China

${ }^{2}$ State Key Laboratory for Strength and Vibration of Mechanical Structure,

School of Aerospace Engineering, Xi'an Jiaotong University, Xi'an 710049,

China

Full list of author information is available at the end of the article. elastomers $^{9}$, electrostrictive polymers ${ }^{10}$, and dielectric elastomers $^{5}$. However, the high voltage needed for actuation and the poor mechanical reliability hinder developments in practical applications. Improving the dielectric constant and lowering the elastic modulus of the polymer dielectrics are efficient ways of lowering the actuating voltage $\mathrm{e}^{11,12}$. Conductive particles or high-k ceramics have been filled into elastomers to increase their dielectric constants, but these rigid fillers have also dramatically increased the elastic modulus and reduced the extensibility ${ }^{13-16}$. Another approach is to use fluids as fillers; liquid-metal microdroplets have been integrated into elastomer substrates, which increased the dielectric constant by over $400 \%$ and avoided the internal compliance mismatch of rigid fillers ${ }^{17}$.

However, polymer dielectrics with optical transmittance functionality are emerging materials with practical significance in next-generation flexible displays and flexible touchscreen panels. By fabricating graphene interlayers to form polymer/graphene/polymer structures, Kim et al. prepared flexible and transparent dielectric films with a high dielectric constant $(\varepsilon)$ of 51 and a transmittance of $\sim 90 \%{ }^{18}$. Park et al. used ultralong metal nanofibers as

\section{(c) The Author(s) 2018}

(c) (i) Open Access This article is licensed under a Creative Commons Attribution 4.0 International License, which permits use, sharing, adaptation, distribution and reproduction in any medium or format, as long as you give appropriate credit to the original author(s) and the source, provide a link to the Creative Commons license, and indicate if changes were made. The images or other third party material in this article are included in the article's Creative Commons license, unless indicated otherwise in a credit line to the material. If material is not included in the article's Creative Commons license and your intended use is not permitted by statutory regulation or exceeds the permitted use, you will need to obtain permission directly from the copyright holder. To view a copy of this license, visit http://creativecommons.org/licenses/by/4.0/. 
A

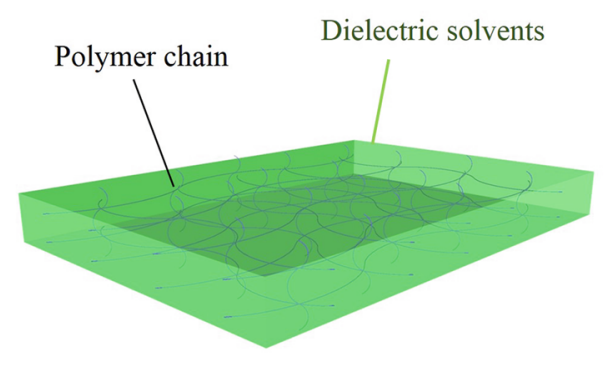

C

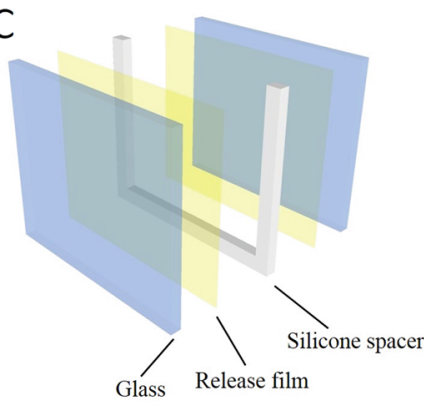

Mold Assembling

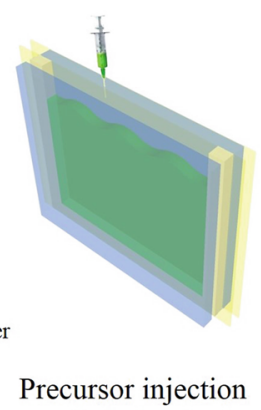

UV light irradiation
B

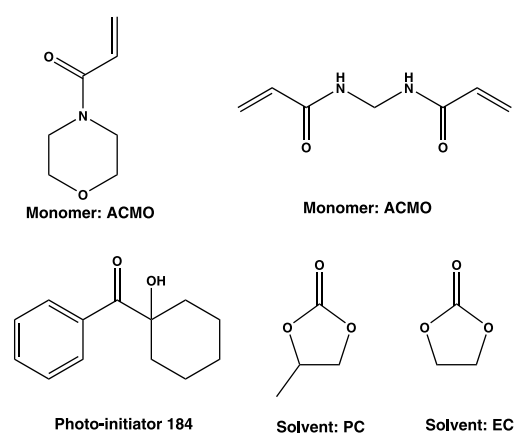

D

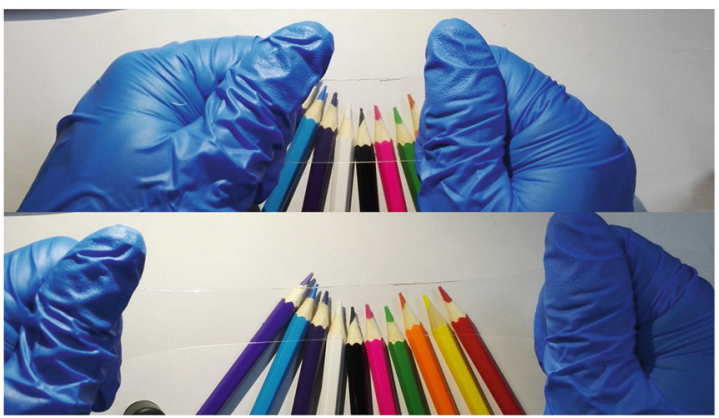

Fig. 1 Basic design and fabrication process of the dielectric gels. a Schematic of a dielectric gel. Both the polymer chain and the solvents in the gel system are dielectric materials. b Ingredients: monomer, 4-acryloylmorpholine (ACMO); cross-linker $N$, $N^{\prime}$-methylenebis (acrylamide) (MBA); photoinitiator, 1-hydroxycyclohexyl phenyl ketone; and solvents, propylene carbonate (PC) and ethylene carbonate (EC). c Fabrication process of a dielectric gel. The gel was synthesized in minutes by photoinitiated polymerization. $\mathbf{d}$ Photographs of the dielectric gels demonstrating good stretchability and high transparency. The upper photograph is the gel without stretching and the lower photograph is the stretched gel

fillers to increase $\varepsilon$ and obtained flexible and transparent dielectric cellulose nanofiber films ( $\varepsilon$ above 9.2 with a high transmittance of $90 \%)^{19}$.

Herein, we introduce a new type of polymer dielectric, dielectric gels. The new materials achieve a unique combination of ultra-high $\varepsilon(30-50)$, low elastic modulus (from 20 to $60 \mathrm{KPa}$ ), and excellent transparency ( 99\%). A gel is a polymer composite with a three-dimensional polymer network that contains a large amount of solvent $^{20,21}$. Gels are present as solid-state soft materials. We designed dielectric gels by using solvents with ultra-high $\varepsilon$ and a polymer network that matched well with the solvents. Dielectric gels offer new opportunities for soft robotics, sensors, electronics, optics, and biomimetics.

\section{Materials and methods}

\section{Synthesis of the dielectric gels}

We mixed appropriate amounts of 4-acryloylmorpholine (ACMO, monomer), $N, N^{\prime}$-methylenebis(acrylamide) (MBA, cross-linker), and 1-hydroxycyclohexyl phenyl ketone (photoinitiator 184) with solvents to form gelation precursor solutions. Unless otherwise stated, the solvent content was fixed at $50 \mathrm{wt} \%$. The precursor solution was poured into a transparent glass mold with a silicone spacer and release films. After irradiation with ultraviolet light
(365 nm, $400 \mathrm{~W}$ power) for $5 \mathrm{~min}$, the dielectric gel was formed. We controlled the thickness of the dielectric gel by adjusting the thickness of the silicone spacer. Dielectric gels for electroactive actuation and tunable lens experiments were synthesized by a similar photocuring process. The solvent ( $\mathrm{PC}: \mathrm{EC}=1: 4$ ) content was fixed at $20 \mathrm{wt} \%$, the volume ratio of 4-acryloylmorpholine (ACMO) and 2ethylhexyl acrylate (2-EHA) monomers was fixed at 1:1, and the molar ratios of the photoinitiator and monomer and the cross-linker and monomer were fixed at $1 \%$ and $0.1 \%$, respectively.

\section{Mechanical tests}

Mechanical tests were performed on an electronic tensile machine (CMT6503, MTS) with a $500 \mathrm{~N}$ load cell. The gels were cut into dumbbell shapes (testing dimensions of $12.0 \times 2.0 \times 2.0 \mathrm{~mm}^{3}$ ) for the tests.

\section{Dielectric tests}

Dielectric tests were performed on a broadband dielectric/impedance spectrometer (Novocontrol GmbH). The gels were 1-mm thick, the testing cupper electrodes were $30 \mathrm{~mm}$ in diameter, and the testing $V_{\text {rms }}$ (Volt root mean square) was set at $1 \mathrm{~V}$. The gel samples were treated without metal sputtering on their surfaces. 

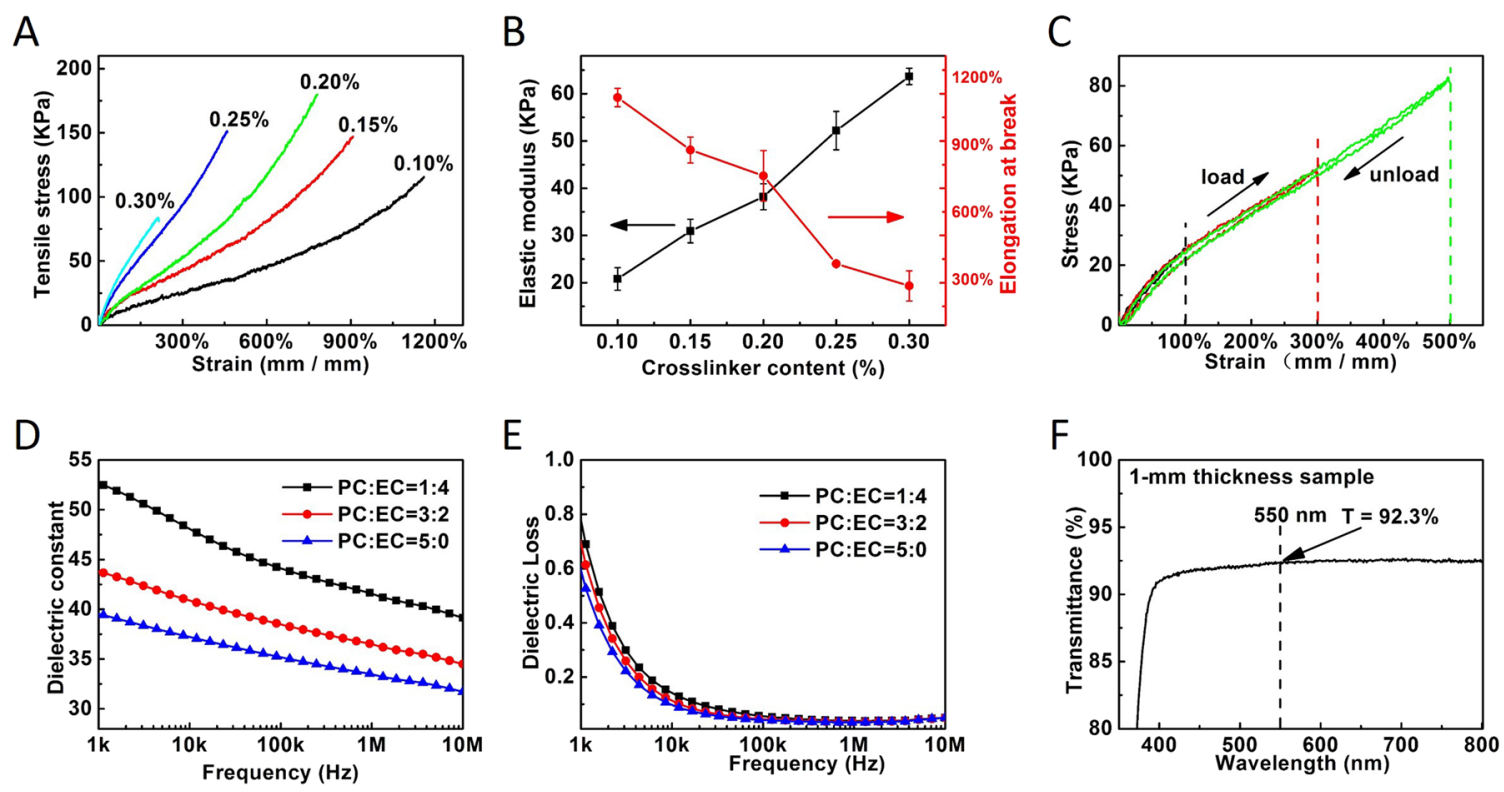

Fig. 2 Performance of the synthesized dielectric gels. The polymer and solvent contents of the testing gels are fixed at 50 wt $\%$ each. a Stress-strain curves of dielectric gels with different cross-linker contents (molar ratios to monomer, as labeled). Each gel was stretched until rupture. b Elastic modulus (calculated from the stress-strain curves at a strain of 10\%) and strain at break, plotted against the cross-linker content. c Cyclic loading of a dielectric gel (cross-linker content of $0.15 \%$ ). The initial loading cycle was set to $100 \%$ strain. After the loading-unloading cycle, the sample was immediately reloaded to $300 \%$ strain for the second cycle and then $500 \%$ strain for the third cycle. $\mathbf{d}$ Plot of the dielectric constants versus testing frequency for the dielectric gels with PC and EC/PC solvents; the ratio represents the volume ratio. e Plot of the dielectric dissipation factor versus testing frequency for the dielectric gels with PC and EC/PC solvents. $\mathbf{f}$ Transmittance test of a 1-mm-thick dielectric gel in the visible range

\section{Transparency tests}

The transparency tests were performed on a ultraviolet-visible spectrophotometer (UV-vis) spectrophotometer (PE Lambda950, Instrument Analysis Center of Xi'an Jiaotong University). The gels for the tests were 1mm thick.

\section{Lens fabrication}

The lenses were fabricated from two gel membranes with silicone oil sealed between them. The membranes were equally biaxially pre-stretched by $\lambda=2.5$ with an original thickness $h=1 \mathrm{~mm}$.

\section{Results}

We chose propylene carbonate (PC) and ethylene carbonate (EC) as solvents for the dielectric gels (Fig. 1a) because they possess ultra-high $\varepsilon$ (65 for PC and 90 for $\mathrm{EC})^{22}$, good chemical stability, low vapor pressure, and low toxicity ${ }^{23}$. They are widely used as electrolyte solvents in lithium-ion batteries. The melting points of $\mathrm{PC}$ and $\mathrm{EC}$ are $-48.8^{\circ} \mathrm{C}$ and $36.4{ }^{\circ} \mathrm{C}$, respectively ${ }^{24}$. Although EC is icy at room temperature, it can be mixed with PC to form a homogeneous solution. The mixed solution is liquid at room temperature and has a higher $\varepsilon$ than $\mathrm{PC}$. We mixed appropriate amounts of 4-acryloylmorpholine (ACMO, monomer), $N, N^{\prime}$-methylenebis (acrylamide) (MBA, cross- linker), and 1-hydroxycyclohexyl phenyl ketone (photoinitiator 184) with the solvents to form gelation precursor solutions (Fig. 1b). Unless otherwise stated, the solvent content was fixed at $50 \mathrm{wt} \%$. The precursor solution was poured into a transparent glass mold with a silicone spacer and release films. After irradiation with ultraviolet light ( $365 \mathrm{~nm}, 400 \mathrm{~W}$ power) for $5 \mathrm{~min}$, the dielectric gel was formed (Fig. 1c, d).

The gels were then cut into dumbbell shapes with dimensions $12.0 \times 2.0 \times 2.0 \mathrm{~mm}^{3}$. Mechanical tests were performed on an electronic tensile machine with a $500 \mathrm{~N}$ load cell. The stretching rate was set at $100 \mathrm{~mm} \mathrm{~min}^{-1}$ during both loading and unloading. The gels possess a low elastic modulus (tens of $\mathrm{KPa}$ ) and good stretchability (with elongation at break ranging from $\sim 200$ to $\sim 1100 \%$ ), as shown in Fig. 2a, b. The cross-linker content of a gel greatly affects its mechanical properties: as the crosslinker content increases, the elastic modulus of a gel increases and the elongation at break decreases. A poly(4acryloylmorpholine) gel with a cross-linker content of $0.15 \%$ was chosen for the cyclic loading-unloading tests (Fig. 2c). The initial loading cycle was set with a strain of $100 \%$. After the loading-unloading cycle, the sample was immediately reloaded to $300 \%$ strain and then to $500 \%$ strain. The gel showed negligible mechanical hysteresis at each strain cycle, and it fully recovered its original length 
after unloading. A long-term cyclic loading-unloading test was also performed, in which the strain was set to $400 \%$; the 100th cycle curve almost coincided with that of the initial cycle, and the elastic modulus remained unchanged (Figure S1).

Dielectric properties were tested on a broadband dielectric/impedance spectrometer. The testing samples were 1-mm thick, and the testing copper electrodes were $30 \mathrm{~mm}$ in diameter. The gel samples were soft enough to be in good contact with the electrodes. The testing $V_{\text {rms }}$ was set at $1 \mathrm{~V}$. Figure $2 \mathrm{~d}$, e shows the dielectric properties of gels with solvents of different $\mathrm{EC} / \mathrm{PC}$ volumetric ratios. The gels exhibit a high $\varepsilon(30 \sim 50)$ over a broad frequency range $(1 \mathrm{~K}-10 \mathrm{MHz})$. The solvent composition greatly affects the $\varepsilon$ of the gels. A higher EC content results in a higher $\varepsilon$. As the frequency increases, the $\varepsilon$ decreases slightly, as shown in Fig. 2d. This may be because the switching of the small-molecule dipoles is unable to match the switching of the electric field at high frequencies. The dielectric loss (loss tangent) of the gels was maintained at $<0.1$ over a frequency range of $20 \mathrm{~K}-10$ $\mathrm{MHz}$. At low frequencies $(10-20 \mathrm{KHz})$, as the frequency decreases, the dielectric loss increases sharply (Figure S2), while the $\varepsilon$ does not change much (Figure S3). We used pure $\mathrm{PC}$ as the solvent and studied the dielectric properties of gels with varying polymer contents of $50,55,60$, and $65 \%$. As the polymer content increases, $\varepsilon$ decreases, and the dielectric loss at low frequency decreases substantially (Figures S4, S5).

The transmittance was measured on an UV-vis spectrophotometer. The gels are highly transparent in the visible range, as shown in Fig. 2f. A sample with a thickness of 1 $\mathrm{mm}$ showed a transmittance of $92.3 \%$ at $550 \mathrm{~nm}$, corresponding to a transmittance of $99.2 \%$ for a $100-\mu \mathrm{m}$-thick gel. The transparency was calculated from the Lambert-Beer equation $\log (T)=K I$, where $T$ is the transparency, $I$ is the thickness of the sample, and $K$ is a constant.

Figure 3a plots the elastic modulus versus $\varepsilon$ for various dielectric elastomers. Our dielectric gels possess a very low elastic modulus and ultra-high $\varepsilon$. The diagram of transmittance versus $\varepsilon$ for different transparent materials is shown in Fig. 3b. We labeled the thickness of the materials for comparison in Fig. $3 \mathrm{~b}$ and show the original transparency of the 1-mm-thick dielectric gel. We also show the transparency of our 0.1-mm-thick dielectric gel calculated according to the Lambert-Beer law. The dielectric gels showed very high transparency and high $\varepsilon$.

We demonstrated the use of the dielectric gel as an electroactive actuator. Figure 4a shows the voltage-induced deformation of the dielectric gel and VHB 4905 (3 M Company), the most frequently used material worldwide with a relative $\varepsilon$ of $\sim 4$ and a shear modulus of a few tens of $\mathrm{KPa}$. A voltage-induced $2200 \%$ areal actuation strain has been achieved, and many functional devices have been

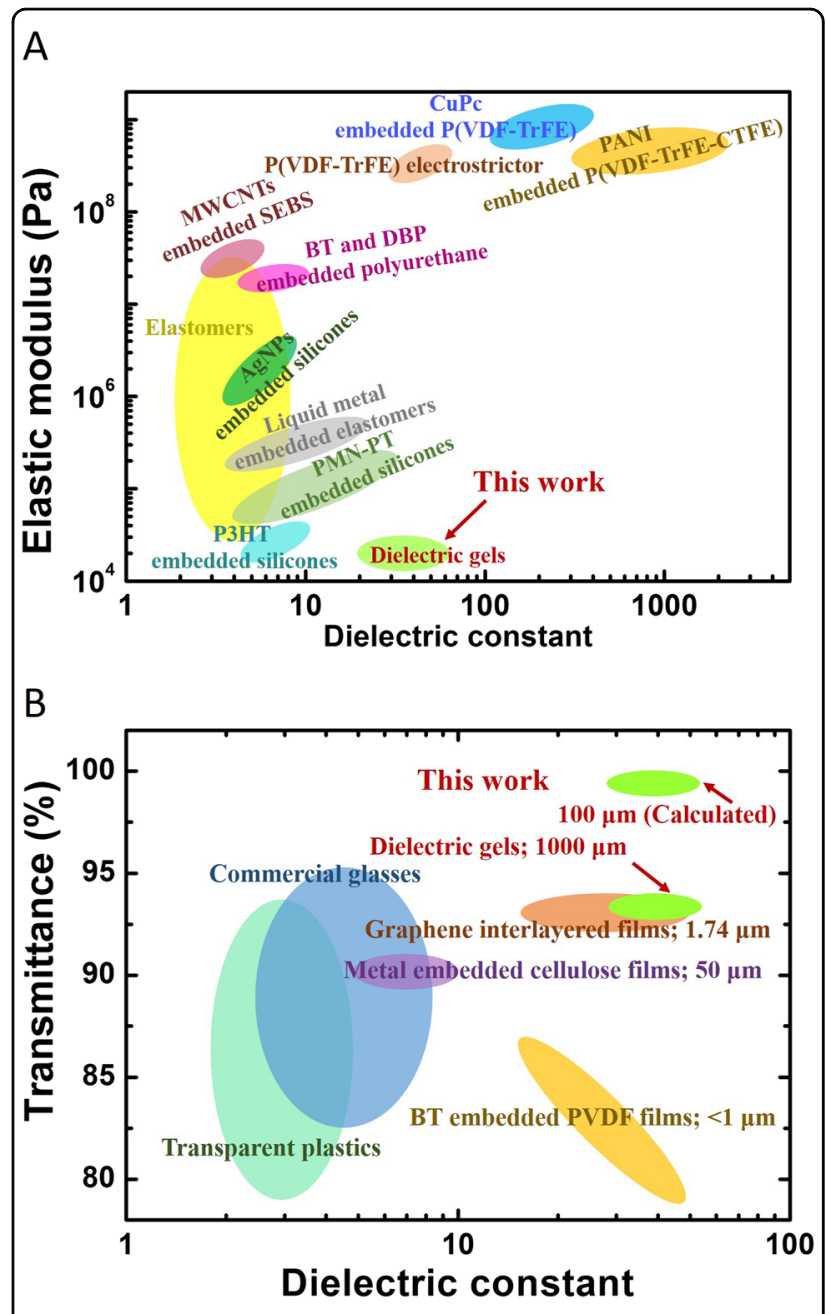

Fig. 3 a Dielectric constant (testing frequency, $1 \mathrm{KHz}$ ) plotted against elastic modulus for dielectric gels, elastomers ${ }^{11}$, lead magnesium niobate-lead titanate (PMN-PT)-embedded silicones ${ }^{13}$, poly(3hexylthiophene) (P3HT)-embedded silicones ${ }^{14}$, multiwalled carbon nanotube (MWCNT)-embedded poly(styrene-b-(ethylene-cobutylene)-b-styrene) (SEBS) ${ }^{15}$, silver nanoparticle (AgNP)-embedded silicones $^{16}$, liquid-metal-embedded elastomers ${ }^{17}$, barium titanate (BT) nanoparticle- and dibutyl phthalate (DBP)-embedded polyurethane ${ }^{29}$, P(VDF-TrFE) electrostrictor ${ }^{10}$, CuPc-embedded P(VDF-TrFE) ${ }^{7}$, and PANIembedded P(VDF-TrFE-CTFE) ${ }^{30}$. b Dielectric constant (testing frequency, $100 \mathrm{KHz}$ ) plotted against transparency for dielectric gels (100- $\mu \mathrm{m}$ thick, this work), glass, transparent plastics, metal-embedded cellulose films $^{31}$, graphene-interlayered films ${ }^{28}$, and BT-embedded poly(vinyldene fluoride) (PVDF) films ${ }^{30}$

demonstrated using $\mathrm{VHB}^{25-27}$. The dielectric gel and VHB material originally had diameter $D=40 \mathrm{~mm}$ and thickness $h=0.5 \mathrm{~mm}$, and both were equally biaxially pre-stretched 1.5 times by 18 clamps. Subsequently, a circular area at the center of the membrane was coated with carbon grease on both sides to act as electrodes. The effective actuation area was actuated from 25 to $28 \mathrm{~mm}$ with a linear strain of $12 \%$ (Supplementary Movie 1). The voltage needed for the dielectric gel is $2000 \mathrm{~V}$, which is much lower than that for 
A

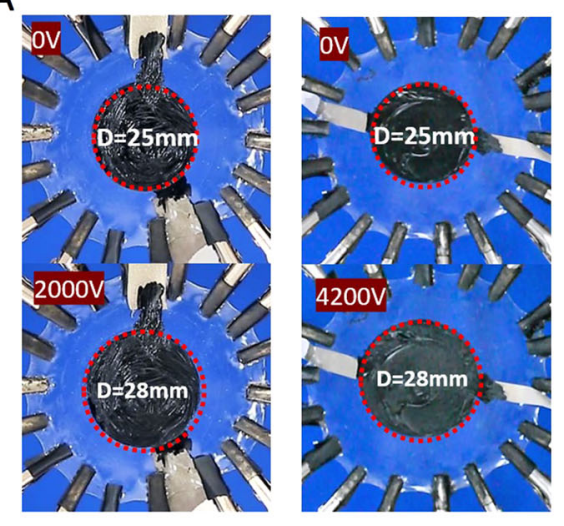

C

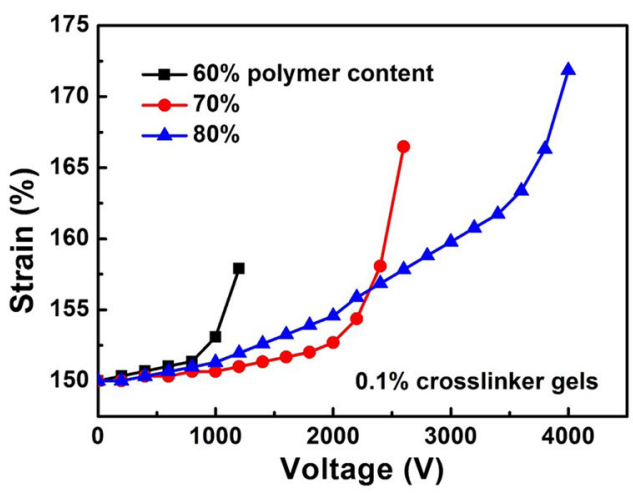

B

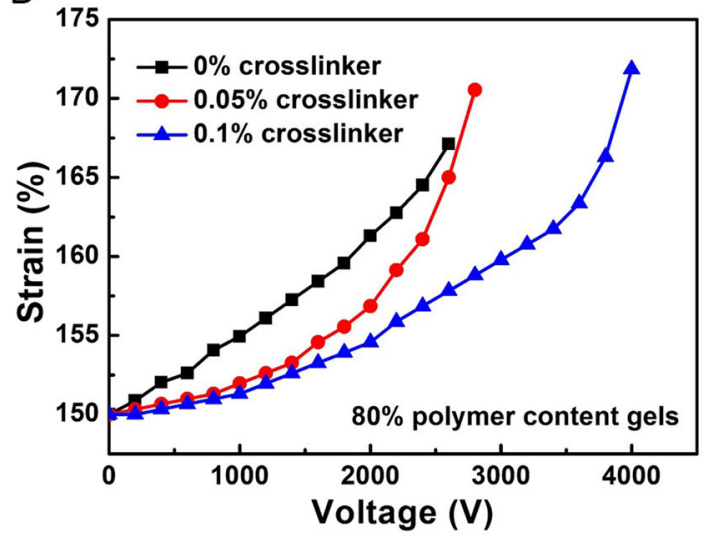

D

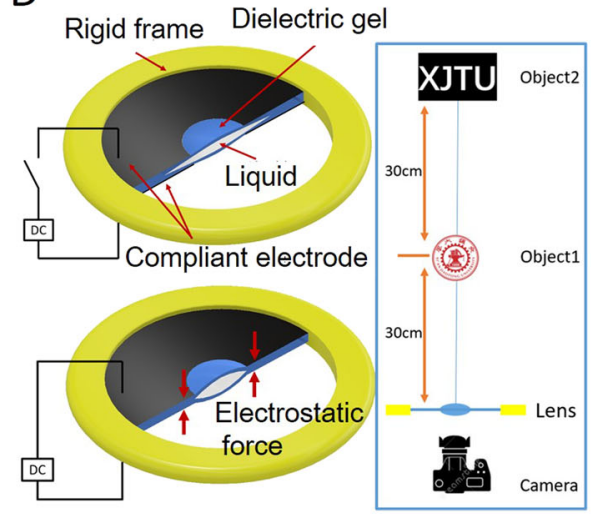

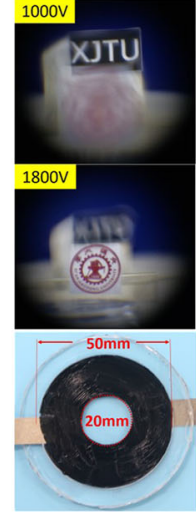

Fig. 4 a The voltage-induced deformation of the dielectric gel (left) and VHB 4905 (right). The initial diameter and thickness are the same. b Strain amplitude as a function of the applied voltage measured at room temperature for dielectric gels with 80\% polymer content. c Strain amplitude as a function of the applied voltage measured at room temperature for dielectric gels with different polymer contents. $\mathbf{d}$ Demonstration of a bioinspired tunable lens made of dielectric gel. The focal length of the lens can be adjusted by varying the applied voltage

VHB (4200 V). Figure 4b, c shows the relationship between the electric field and the areal actuation strain. The gels for the electroactuation tests were both equally biaxially prestretched 1.5 times by 18 clamps. Subsequently, a circular area at the center of the membrane was coated with carbon grease on both sides to act as electrodes. We used a camera to catch the effective actuation area as the applied voltage increased until breakdown. The ordinate values were the linear strain. In Fig. 4b, we fixed the polymer content of the gels at $80 \%$, and as the cross-linker content increased, the maximum actuation strain increased. At a fixed actuation strain, the needed voltage increases, which may be due to the higher elastic modulus of the gels at higher cross-linker content. Figure 4c shows the electroactuation properties of gels with different polymer contents. At higher polymer content, the maximum actuation strain was higher, and high voltages were difficult to sustain for gels with high liquid content (low polymer content). Figure 4d shows a demonstration of a voltage-driven tunable lens made of dielectric gels, referring to a similar configuration ${ }^{28}$.
Transparent liquid was sandwiched between two membranes composed of the dielectric gels. The physical properties, including the mechanical and dielectric properties, of the dielectric gels used for electroactive actuation tests are shown in Figures S6, S7. When a voltage is applied, the two membranes tend to close and squeeze the liquid in the center, resulting in a change in the focal length of the biomimic lens. One can clearly resolve an object near the lens or an object far away from the lens by varying the applied voltage (Fig. 4d, Supplementary Movie 2). The design of the lens is inspired by human eyes. For human eyes, the ciliaris contracts to change the focal length of the eye. In our design, the dielectric gel acts as the muscle. When the dielectric gel is subjected to a voltage, it contracts to change the focal length of the lens.

\section{Discussion}

In conclusion, by using high $\varepsilon$ solvents, i.e., $\mathrm{PC}$ and $\mathrm{EC} / \mathrm{PC}$ mixtures, we designed and fabricated a series of high-performance dielectric gels that simultaneously 
displayed ultra-high $\varepsilon$ (30-50), low elastic modulus (from 20 to $60 \mathrm{KPa}$ ), and excellent transparency. The dielectric gels showed high stretchability (stretch of $\sim 10$ ) and low mechanical hysteresis. We demonstrated that the voltage needed for the same actuation strain is reduced by a half using our dielectric gels, which are electroactive materials, compared with the most commonly used dielectric elastomer VHB. We demonstrated the use of the dielectric gel by fabricating a bioinspired tunable lens, the focal length of which can be adjusted by varying the applied voltage. As a new type of polymer dielectric, we believe that these dielectric gels offer new opportunities for soft robotics, sensors, electronics, optics, and biomimetics.

\section{Acknowledgements}

This research was supported by the National Natural Science Foundation of China (Nos. 51773165, 11772249), the Fundamental Research Funds for the Central Universities (xjj2015119), and the Science Technology Innovation Commission of Shenzhen Municipality (JCYJ 20170817171930009). We thank Mr. Junjie Zhang, Ms. Axin Lu (Instrument Analysis Center of Xi'an Jiaotong University), and Ms. Jingjing Liu for their valuable assistance during testing.

\section{Author details}

'Department of Applied Chemistry, School of Science, MOE Key Laboratory for Nonequilibrium Synthesis and Modulation of Condensed Matter, State Key Laboratory for Mechanical Behavior of Materials, Xi'an Jiaotong University, Xi'an 710049, China. ${ }^{2}$ State Key Laboratory for Strength and Vibration of Mechanical Structure, School of Aerospace Engineering, Xi'an Jiaotong University, Xi'an 710049, China. ${ }^{3}$ The First Affiliated Hospital of Shenzhen University, Health Science Center, Shenzhen 518028, China

\section{Authors' contributions}

L.S., R.Y., S.L., T.L., and S.D. designed the study and interpreted the results. L.S. developed the protocol for fabrication of the gels and prepared the initial samples. R.Y. designed and performed the electroactive actuation tests and tunable lens tests. T.W. and W.W. contributed to the discussion of the results. L.S., T.L., and S.D. wrote the manuscript. All authors commented on the manuscript.

\section{Conflict of interest}

The authors declare that they have no conflict of interest.

\section{Publisher's note}

Springer Nature remains neutral with regard to jurisdictional claims in published maps and institutional affiliations.

Supplementary Information is available for this paper at https://doi.org/ 10.1038/s41427-018-0077-7.

Received: 26 March 2018 Revised: 14 June 2018 Accepted: 25 June 2018. Published online: 27 August 2018

\section{References}

1. Chu, B. et al. A dielectric polymer with high electric energy density and fast discharge speed. Science 313, 334-336 (2006).

2. Mannsfeld, S. C. B. et al. Highly sensitive flexible pressure sensors with microstructured rubber dielectric layers. Nat. Mater. 9, 859-864 (2010).

3. Naber, R. C. G. et al. High-performance solution-processed polymer ferroelectric field-effect transistors. Nat. Mater. 4, 243-248 (2005).

4. $\mathrm{Ma}$, R. J. et al. Highly efficient electrocaloric cooling with electrostatic actuation. Science 357, 1130-1134 (2017).

5. Pelrine, R., Kornbluh, R., Pei, Q. B. \& Joseph, J. High-speed electrically actuated elastomers with strain greater than 100\%. Science $\mathbf{2 8 7}$, 836-839 (2000).
6. Carpi, F., Bauer, S. \& De Rossi, D. Stretching dielectric elastomer performance. Science 330, 1759-1761 (2010).

7. Zhang, Q. M. et al. An all-organic composite actuator material with a high dielectric constant. Nature 419, 284-287 (2002).

8. Gao, Q. \& Scheinbeim, J. I. Dipolar intermolecular interactions, structural development, and electromechanical properties in ferroelectric polymer blends of nylon-11 and poly(vinylidene fluoride). Macromolecules 33, 7564-7572 (2000).

9. Lehmann, W. et al. Giant lateral electrostriction in ferroelectric liquid-crystalline elastomers. Nature 410, 447-450 (2001).

10. Zhang, Q. M., Bharti, V. \& Zhao, X. Giant electrostriction and relaxor ferroelectric behavior in electron-irradiated poly(vinylidene fluoride-trifluoroethylene) copolymer. Science 280, 2101-2104 (1998).

11. Brochu, P. \& Pei, Q. Advances in dielectric elastomers for actuators and artificial muscles. Macromol. Rapid Commun. 31, 10-36 (2010).

12. Madsen, F. B., Daugaard, A. E., Hvilsted, S. \& Skov, A. L. The current state of silicone-based dielectric elastomer transducers. Macromol. Rapid Commun. 37, 378-413 (2016).

13. Gallone, G., Carpi, F., De Rossi, D., Levita, G. \& Marchetti, A. Dielectric constant enhancement in a silicone elastomer filled with lead magnesium niobate-lead titanate. Mater. Sci. Eng. C Biomim. Supramol. Syst. 27, 110-116 (2007).

14. Carpi, F., Gallone, G., Galantini, F. \& De Rossi, D. Silicone-poly(hexylthiophene) blends as elastomers with enhanced electromechanical transduction properties. Adv. Funct. Mater. 18, 235-241 (2008).

15. Yadav, S. K. Mahapatra, S. S., Cho, J. W. \& Lee, J. Y. Functionalization of multiwalled carbon nanotubes with poly(styrene-b-(ethylene-co-butylene)-bstyrene) by click coupling. J. Phys. Chem. C 114, 11395-11400 (2010).

16. Quinsaat, J. E. Q. et al. Highly stretchable dielectric elastomer composites containing high volume fractions of silver nanoparticles. J. Mater. Chem. A $\mathbf{3}$, 14675-14685 (2015)

17. Bartlett, M. D. et al. Stretchable, high-k dielectric elastomers through liquidmetal inclusions. Adv. Mater. 28, 3726-3731 (2016).

18. Kim, J.-Y. et al. Flexible and transparent dielectric film with a high dielectric constant using chemical vapor deposition-grown graphene interlayer. ACS Nano 8, 269-274 (2014)

19. Ji, S. et al. High dielectric performances of flexible and transparent cellulose hybrid films controlled by multidimensional metal nanostructures. Adv. Mater. https://doi.org/10.1002/adma.201700538 (2017).

20. Kamata, H., Akagi, Y., Kayasuga-Kariya, Y., Chung, U. \& Sakai, T. "Nonswellable" hydrogel without mechanical hysteresis. Science 343, 873-875 (2014).

21. Sun, J.-Y. et al. Highly stretchable and tough hydrogels. Nature 489, 133-136 (2012).

22. Payne, R. \& Theodorou, I. E. Dielectric properties and relaxation in ethylene carbonate and propylene carbonate. J. Phys. Chem. 76, 2892-2900 (1972).

23. Schaffner, B., Schaffner, F., Verevkin, S. P. \& Borner, A. Organic carbonates as solvents in synthesis and catalysis. Chem. Rev. 110, 4554-4581 (2010).

24. $\mathrm{Xu}, \mathrm{K}$. Nonaqueous liquid electrolytes for lithium-based rechargeable batteries. Chem. Rev. 104, 4303-4417 (2004).

25. An, L., Wang, F. F., Cheng, S. B., Lu, T. Q. \& Wang, T. J. Experimental investigation of the electromechanical phase transition in a dielectric elastomer tube. Smart Mater. Struct. 24, https://doi.org/10.1088/0964-1726/24/3/035006 (2015).

26. Anderson, I. A., Gisby, T. A., McKay, T. G., O'Brien, B. M. \& Calius, E. P. Multifunctional dielectric elastomer artificial muscles for soft and smart machines. J. Appl. Phys. 112, https://doi.org/10.1063/1.4740023 (2012).

27. Lu, T. Q., Shi, Z. B., Shi, Q. \& Wang, T. J. Bioinspired bicipital muscle with fiberconstrained dielectric elastomer actuator. Extreme Mech. Lett. 6, 75-81 (2016).

28. Carpi, F., Frediani, G., Turco, S. \& De Rossi, D. Bioinspired tunable lens with muscle-like electroactive elastomers. Adv. Funct. Mater. 21, 4152-4158 (2011).

29. Yin, G. et al. Dielectric elastomer generator with improved energy density and conversion efficiency based on polyurethane composites. ACS Appl. Mater. Interfaces 9, 5237-5243 (2017).

30. Huang, C., Zhang, Q. M. \& Su, J. High-dielectric-constant all-polymer percolative composites. Appl. Phys. Lett. 82, 3502-3504 (2003).

31. Kamezawa, N., Nagao, D., Ishii, H. \& Konno, M. Transparent, highly dielectric poly(vinylidene fluoride) nanocomposite film homogeneously incorporating BaTiO3 nanoparticles with fluoroalkylsilane surface modifier. Eur. Polym. J. 66, 528-532 (2015) 